\title{
Analyses of prognostic factors in cases of non-small cell lung cancer with multiple brain metastases
}

\author{
This article was published in the following Dove Press journal: \\ OncoTargets and Therapy \\ I March 2016 \\ Number of times this article has been viewed
}

\author{
Xiaomei Gong' \\ Daoan Zhou' \\ Shixiong Liang' \\ Caicun Zhou ${ }^{2}$ \\ 'Department of Radiation Oncology, \\ 2Department of Oncology, Shanghai \\ Pulmonary Hospital, Tongji University \\ School of Medicine, Shanghai, People's \\ Republic of China
}

Aim: To observe the therapeutic efficacy and prognostic factors that influence survival rates in non-small cell lung cancer (NSCLC) patients with multiple brain metastases (BMs), (more than three and less than ten).

Methods: Retrospective analyses were conducted on the clinical data of 209 NSCLC patients with multiple BMs and were admitted to our hospital between March 2007 and November 2012. All BM patients received whole-brain radiotherapy. Two hundred patients received combined chemotherapy during the treatment process; 99 received targeted drug therapy; and nine got only symptomatic and supportive treatment. Survival time was defined as the period from the start of BM therapy to the patient's death or end of the follow-up period. The Kaplan-Meier method was used to calculate the median survival time, and the 6-month, 1-, and 2-year cumulative survival rates, as well as to plot the survival curves. The patients' cultural background included their socioeconomic status, level of education, their understanding of the disease, and the degree of care and support they received from their family members. Log-rank test was employed to test the differences in the survival rates between the subgroups. Cox multivariate regression analyses were used to analyze the various factors influencing the prognoses of NSCLC with multiple BMs.

Results: The follow-up duration was between 1 and 87 months. The median survival time for all BM patients was 12.1 months (95\% confidence interval 9.37-14.83). The 6-month, 1-, and 2-year cumulative survival rates were $80 \%, 50.2 \%$, and $10.7 \%$, respectively. Univariate analyses revealed that the independent factors influencing survival prognoses included Karnofsky Performance Status score, control of the primary lung tumor, interval between the confirmed diagnoses of lung cancer and BM, presence of extracranial metastasis, number of chemotherapy cycles undergone, Graded Prognostic Assessment class, administration of combined targeted drug therapy, the pathological type, and the cultural backgrounds of the patients. Multivariate analyses indicated that the number of chemotherapy cycles, administration of combined drug therapy, and patients' cultural background were key independent factors influencing survival prognoses.

Conclusion: Active treatment of NSCLC with multiple BMs was beneficial, and the patients' cultural background had a strong influence on survival prognoses. Chemotherapy combined with targeted drug therapy could increase the patients' median and overall survival rates. The number of chemotherapy cycles undergone, administration of combined targeted drug therapy, and the cultural backgrounds of the patients had significant effects on the patients' survival prognoses. Keywords: cancer, non-small cell lung cancer, brain tumor, whole-brain radiotherapy, targeted drug therapy, cultural background

\section{Introduction}

The incidence of non-small cell lung cancer (NSCLC) has gradually increased in recent years. It is currently the most common malignant cancer, and its incidence of brain metastasis (BM) is up to $30 \%-55 \% .{ }^{1}$ Since the 1970 s, radiotherapy has become the standard treatment for BM, and is able to increase the median survival time to 
4 months. ${ }^{2,3}$ The recent improvements in platinum-based agents, the emergence of targeted drugs, as well as the developments in research on the blood-brain barrier have enabled us to achieve a certain level of therapeutic efficacy in chemotherapy and biotherapy for lung cancer BM., ${ }^{4,5}$ Studies have reported that the median survival time for NSCLC metastasis treated using chemotherapy combined with radiotherapy was 6-12 months, ${ }^{6,7}$ while that of patients treated using targeted drug therapy combined with radiotherapy was 11-23 months. ${ }^{8,9}$ Studies have also shown that the socioeconomic status and education level of the patients could influence their survival time; specifically, lower socioeconomic status and education level correlated with poorer prognosis in cancer patients. ${ }^{10,11}$ Two hundred and nine NSCLC patients with BM were admitted and treated in our hospital between March 2007 and November 2012. The clinical data for these patients were collated, and then analyzed for their survival status and the main factors influencing prognosis.

\section{Data and methods}

\section{Clinical data}

A total of 209 NSCLC patients with BM who received whole-brain radiotherapy (WBRT) in our hospital between March 2007 and November 2012 were selected. The diagnosis for all 209 cases of lung cancer patients was based on either cytological or histological evidence. The conditions of patients with BM included: the number of BM tumors is more than three and less than ten, all tumors are less than $5 \mathrm{~cm}$ in diameter. Exclusion of cerebellum placeholder and meningeal metastases. BM was verified using magnetic resonance imaging or computed tomography. Among these patients, there were 148 cases of adenocarcinoma, 42 cases of squamous cell carcinoma, four cases of adenosquamous carcinoma, and 15 cases of NSCLC (without specific histologic subtype). The median age at the time of BM diagnosis was 58 years (range, $31-83$ years) for all patients (Table 1 for details on other factors). The study protocol was approved

Table I Univariate analysis for the prognosis of 209 NSCLC patients with multiple brain metastases

\begin{tabular}{|c|c|c|c|c|}
\hline Factor & Number of cases & Median survival time (months) & $\chi^{2}$ & $P$-value \\
\hline \multicolumn{5}{|l|}{ KPS score } \\
\hline$\geq 70$ points & 185 & 13.1 & 24.96 & 0.000 \\
\hline$<70$ points & 24 & 6 & & \\
\hline \multicolumn{5}{|c|}{ Control of primary lung tumor } \\
\hline Controlled & 42 & 19.83 & 22.72 & 0.000 \\
\hline Progressive & 167 & 9.03 & & \\
\hline \multicolumn{5}{|c|}{ Interval between confirmed diagnosis of lung cancer and brain metastasis } \\
\hline$\geq$ I year & 45 & 19.67 & 17.18 & 0.000 \\
\hline$<$ I year & 164 & 8.87 & & \\
\hline \multicolumn{5}{|c|}{ Presence of combined extracranial metastasis } \\
\hline Absent & 37 & 19.73 & 7.12 & 0.008 \\
\hline Present & 172 & 11.3 & & \\
\hline \multicolumn{5}{|c|}{ Number of chemotherapy cycles } \\
\hline$\geq 3$ cycles & 120 & 14.97 & 15.98 & 0.000 \\
\hline$<3$ cycles & 89 & 8.5 & & \\
\hline \multicolumn{5}{|l|}{ GPA class } \\
\hline Class I & 29 & 20.6 & 48.46 & 0.000 \\
\hline Class 2 & 143 & 12.9 & & \\
\hline Class 3 & 37 & 6.1 & & \\
\hline \multicolumn{5}{|c|}{ Presence of combined targeted drug therapy } \\
\hline Absent & 110 & 8.1 & 29.78 & 0.000 \\
\hline Present & 99 & 18.7 & & \\
\hline \multicolumn{5}{|c|}{ Pathological type } \\
\hline \multicolumn{5}{|c|}{ Patient's cultural background } \\
\hline Adenocarcinoma & 148 & 12.9 & 13.82 & 0.000 \\
\hline Squamous & 42 & 9.1 & 349.52 & 0.000 \\
\hline Adenosquamous & 4 & 5.57 & & \\
\hline NSCLC (NOS) & 15 & 7.27 & & \\
\hline Level 0 & 22 & 3.2 & & \\
\hline Level I & 35 & 6.63 & & \\
\hline Level 2 & 87 & 14.27 & & \\
\hline Level 3 & 62 & 19.63 & & \\
\hline Level 4 & 3 & 15.93 & & \\
\hline
\end{tabular}

Abbreviations: GPA, Graded Prognostic Assessment; KPS, Karnofsky Performance Status; NOS, not otherwise specified; NSCLC, non-small cell lung cancer. 
by the Shanghai Pulmonary Hospital ethics committee, and all participants provided written informed consent.

\section{Treatment conditions of primary lung tumor}

Thirty-six patients had previously undergone surgical treatment, and the surgical procedures included pneumonectomy, lobectomy, and palliative tumor resection. One hundred and twenty-three patients had previously undergone thoracic radiotherapy, and the irradiation area included the primary tumor area and/or the lymph node drainage area, while the radiation doses were between 30 and 60 Gy (palliative and definitive radiotherapy). Two hundred patients had received chemotherapy during the treatment process, mainly a combination of cisplatin with other drugs. Among these patients, 99 had undergone targeted therapy. In the 99 patients who underwent targeted therapy, only nine detected the epidermal growth factor receptor (EGFR) mutation status. The patients who did not detect the EGFR mutation status were mostly female (83), did not smoke (90), and had adenocarcinoma (90). In the 90 patients, tyrosine kinase inhibitor (TKI) was only used for the second- or third-line therapy; patients had begun taking the targeted drugs since the occurrence of BM. The targeted drugs included Irressa, which was taken at a dose of $250 \mathrm{mg}$ once daily with or without food. The drugs were taken until tumor progression, which was evaluated using the Response Evaluation Criteria In Solid Tumors. Palliative treatment included antitussives, phlegm relief, analgesics, hemostasis, pleural drainage, and drug injections.

\section{Treatment conditions for BM}

All patients received WBRT. A 6 MV X-ray was used for parallel bilateral irradiation and the total irradiation dose was 3,000 cGy/10 fx. Sixteen patients terminated radiotherapy due to their inability to tolerate the side effects, of whom eight patients received a total dose of 2,100 cGy/7 fx, and eight patients received $900 \mathrm{cGy} / 3 \mathrm{fx}$. General treatment included administration of mannitol and dexamethasone to reduce intracranial pressure, as well as the provision of symptomatic and supportive treatments.

\section{Measurement of outcomes} Short-term efficacy

Evaluation of primary lung tumor and BM after treatment was performed using the Response Evaluation Criteria In Solid Tumors. The sum of the longest diameters for all target lesions was calculated and labeled as the baseline sum of longest diameters. The baseline sum of longest diameters was taken as the main baseline indicator for the evaluation of tumor therapeutic efficacy.

\section{Criteria for therapeutic efficacy}

1. Evaluation of target lesions was performed by measuring and calculating the sum of longest diameter of all target lesions, followed by the comparison with the baseline sum of longest diameters. The objective tumor evaluation criteria were as follows: complete remission - all target lesions have disappeared; partial remission - at least $30 \%$ reduction in the target lesion sum of longest diameters compared with baseline; progressive disease - at least $20 \%$ increase in target lesion sum of longest diameters compared with the smallest target lesion sum of longest diameter recorded since treatment began, or the emergence of one or more new lesions; stable disease - tumor growth between partial remission and disease progression.

2. Long-term therapeutic efficacy: median survival time, and 6-month, 1-, and 2-year cumulative survival rate.

\section{Statistical methods}

Survival rate was calculated with the Kaplan-Meier method. A log-rank test was employed to compare the differences in the survival rate of each subgroup. Multivariate risk factor analysis was performed using the Cox proportional hazards model. Data processing was carried out using SPSS 16.0 (SPSS Inc., Chicago, IL, USA).

\section{Results}

The follow-up time was between 1 and 87 months. The median survival time after treatment for BM was 12.1 months. The 6-month, 1-, and 2-year cumulative survival rates were $80 \%, 50.2 \%$, and $10.7 \%$, respectively. Univariate analysis (Table 1) indicated that favorable prognostic factors included: Karnofsky Performance Status (KPS) scores during BM treatment, control of the primary lung tumor, interval between confirmed diagnosis of lung cancer and BM, presence of combined extracranial metastasis, number of chemotherapy cycles undergone, Graded Prognostic Assessment class, presence of combined targeted therapy, the pathological type, and patients' cultural background. Multivariate analysis (Table 2) indicated that the number of chemotherapy cycles, combined targeted therapy, and patients' cultural background were key prognostic factors influencing survival. The survival curves influenced by these three factors are shown in Figures 1-3, respectively. 
Table 2 Multivariate analyses for the prognosis of 209 NSCLC patients with multiple brain metastases

\begin{tabular}{|c|c|c|c|c|c|c|c|}
\hline Factor & Group & $d f$ & B & SE & Wald & $P$-value & $95 \% \mathrm{Cl}$ \\
\hline Chemotherapy cycles & $<3$ cycles $/ \geq 3$ cycles & I & 0.54 & 0.17 & 10.44 & 0.001 & $|.234-2.36|$ \\
\hline $\begin{array}{l}\text { Presence of targeted } \\
\text { therapy }\end{array}$ & Present/absent & I & 0.41 & 0.16 & 6.68 & 0.01 & $1.103-2.045$ \\
\hline \multirow[t]{5}{*}{ Cultural background } & & 4 & & & 128.84 & 0.000 & \\
\hline & $\begin{array}{l}\text { Illiterate }+ \text { poor understanding of disease }+ \text { poor } \\
\text { family support }\end{array}$ & I & 4.154 & 0.737 & 31.754 & 0.000 & $18.708-330.612$ \\
\hline & $\begin{array}{l}\text { Primary school }+ \text { average understanding of } \\
\text { disease }+ \text { average family support }\end{array}$ & I & 2.512 & 0.664 & 14.318 & 0.000 & $4.209-55.329$ \\
\hline & $\begin{array}{l}\text { Junior secondary school + strong understanding } \\
\text { of disease }+ \text { strong family support }\end{array}$ & I & 0.295 & 0.609 & 0.235 & 0.628 & $0.445-4.805$ \\
\hline & $\begin{array}{l}\text { Senior secondary school + strong understanding } \\
\text { of disease + strong family support } \\
\text { University + very strong understanding of } \\
\text { disease + very strong family support }\end{array}$ & I & -0.239 & 0.612 & 0.153 & 0.696 & $0.237-2.612$ \\
\hline
\end{tabular}

Abbreviations: $\mathrm{Cl}$, confidence interval; NSCLC, non-small cell lung cancer; SE, standard error.

\section{Discussion}

Metastasis of lung cancer accounts for $30 \%-40 \%$ of all $\mathrm{BMs},{ }^{12}$ and occurs most frequently in lung adenocarcinomas. The incidence of BM in adenocarcinoma among the 209 patients in our study was $71 \%$, while that of squamous cell carcinoma was $20 \%$, and that of adenosquamous carcinomas and NSCLC (not otherwise specified) was 9\%. BM mainly occurs via a hematogenous route. As the growth of BM and disease progression is rapid, palliative therapy is the main care provided to patients. WBRT can improve the patients' neurological symptoms, increase their quality

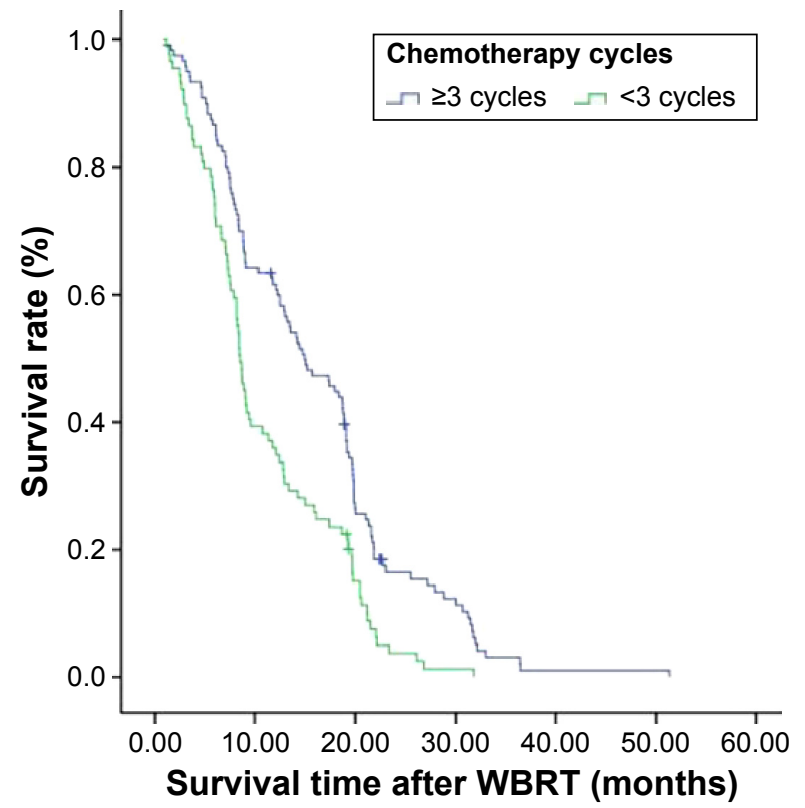

Figure I The effect of different chemotherapy cycles on the survival rate of NSCLC patients with brain metastasis.

Abbreviations: NSCLC, non-small cell lung cancer; WBRT, whole-brain radiotherapy. of life, and adequately prolong survival time; its efficacy is higher than $60 \% .^{13}$ Since the majority of chemotherapy drugs encounter difficulties in passing through the bloodbrain barrier, the therapeutic efficacy of chemotherapy alone is poor. Recent research has shown that WBRT combined with targeted therapy was able to increase patients' median survival time, a finding that was further verified in our retrospective data analysis. This was also influenced by the different cultural backgrounds of the patients.

Radiotherapy is one of the main treatment methods for metastasis of lung cancer to the brain. The median survival

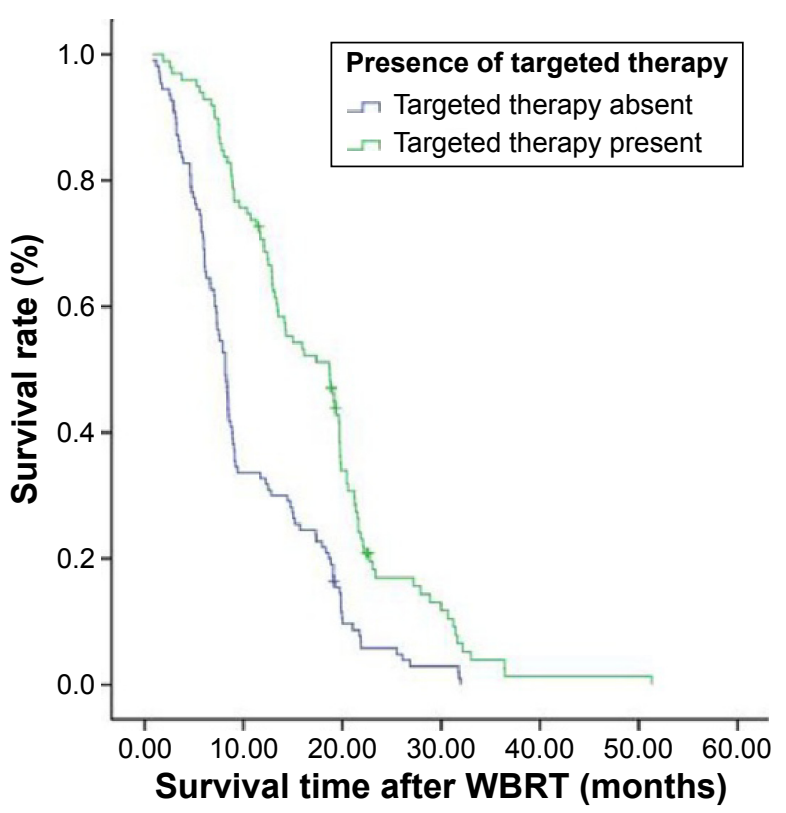

Figure 2 The effect of the presence of combined targeted therapy on the survival rate of NSCLC patients with brain metastasis.

Abbreviations: NSCLC, non-small cell lung cancer; WBRT, whole-brain radiotherapy. 
Cultural background

$\neg$ Illiterate + poor understanding of disease + poor family support

$\neg$ Primary school + average understanding of disease + average family support Junior secondary school + strong understanding of disease + strong family support

$\neg$ Senior secondary school + strong understanding of disease + strong family support University + very strong understanding of disease + very strong family support

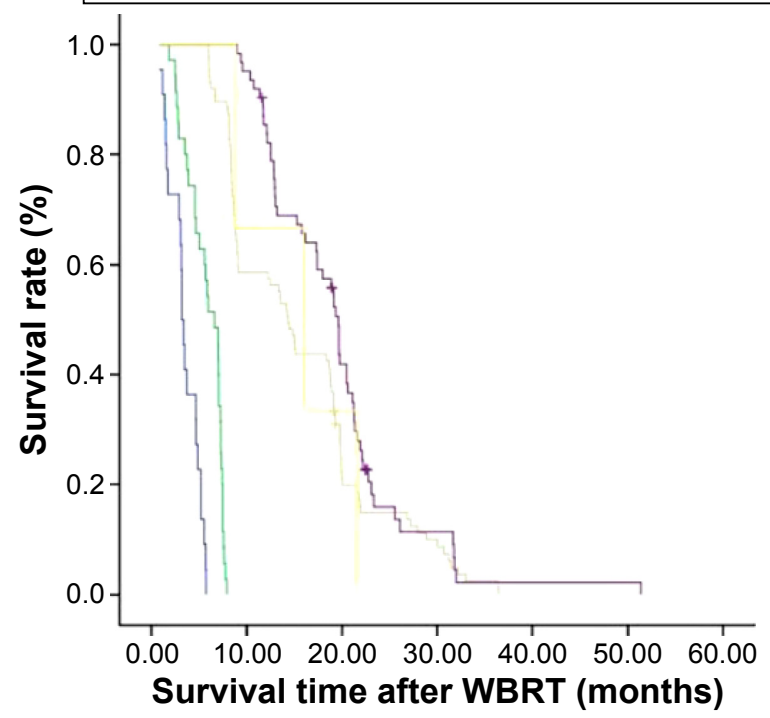

Figure 3 The effect of different cultural backgrounds on the survival rate of NSCLC patients with brain metastasis.

Abbreviations: NSCLC, non-small cell lung cancer; WBRT, whole-brain radiotherapy.

time of patients with BM treated with WBRT alone can be increased by 3-6 months. The aim of WBRT is to eliminate subclinical lesions in order to prevent recurrence. Stereotactic radiosurgery (SRS) now has become more widely used focal treatment modality for patients who have BM. Radiation Therapy Oncology Group 9508 was a randomized, controlled, Phase III trial of 333 patients with one to three BMs and a KPS of 70 or greater who were treated with WBRT and SRS or WBRT alone. ${ }^{14}$ In patients who had a single BM, treatment with WBRT and SRS compared with only WBRT resulted in a decreased rate of local recurrence at 1 year $(18 \%$ vs $29 \% ; P=0.01)$ and superior median survival times ( 6.5 vs 4.9 months; $P=0.039$ ). In patients who had two or three BMs, local control was significantly improved in the combination arm, but there was no difference in survival time between the two groups (5.8 vs 6.7 months; $P=0.097$ ). There was an additional benefit in outcomes (maintenance or improvement of KPS and corticosteroid use) in patients. Our data also suggest that KPS is one of the prognostic factors. Chang et a ${ }^{15}$ performed a Phase III study in patients who had one to three BMs that compared the approach of combination of SRS and WBRT versus SRS alone. The primary endpoint of this study was neurocognitive function, as measured by the Hopkins Verbal Learning Test-Revised. The trial was stopped early after 58 patients were accrued because of a high probability that the SRS-plus-WBRT arm would show a significant decline in learning and memory function at 4 months. SRS plus WBRT is too neurotoxic to choice. Some oncologists have been concerned that WBRT adversely affects neurocognition. ${ }^{16}$ However, a study of 208 patients with BM found that patients who responded after WBRT had improved neurocognitive function and that tumor progression affected neurocognition more than WBRT. ${ }^{17}$ While in a prospective, observational study for one to ten BMs performed in 23 hospitals in Japan, no difference in overall survival was demonstrated in patients who had two to four BMs versus five or more BMs when treated with SRS alone. ${ }^{18}$ The median overall survival after SRS was 13.9 months in patients who had a single BM, 10.8 months in patients who had two to four BMs, and 10.8 months in patients who had five to ten BMs. We know that the study is a single arm trial. There is no WBRT control group, and we could find that it has a high intracranial metastasis after SRS. As the tumor number increases, the rate of intracranial metastases goes higher. In our clinical treatment, we chose WBRT with 30 Gy in ten fractions for BMs with the number more than three and less than ten.

EGFR-TKIs are targeted drugs that have been receiving constant attention in the field of oncology in recent years. EGFR-TKIs have lower side effects than cytotoxic drugs, and have shown a certain degree of therapeutic efficacy in advanced NSCLC. Researchers have found that gefitinib has a low molecular weight, and in vivo $14 \mathrm{C}$ labeling in animal studies revealed that it had relatively good cell penetration. Gefitinib is able to pass through the blood-brain barrier, and its concentration in brain tissue and spinal cord was found to be higher than that of other chemotherapy drugs. ${ }^{19}$ Gefitinib also showed relatively good toxic effects on malignant brain tumors in animal models. ${ }^{20}$ Moreover, recent reports have indicated that gefitinib had a relatively good therapeutic efficacy in the treatment of NSCLC BM. ${ }^{21,22}$

Our data analysis has demonstrated that regardless of whether targeted drug treatment was the first-, second-, or third-line therapy, it had greater efficacy in the treatment of NSCLC patients with BM compared with combined WBRT and cytotoxic chemotherapy. The median survival time for patients using this therapy was 18.7 months, thus using targeted therapy greatly increased the median survival time of 
lung cancer patients with BM. Multivariate analyses in past studies have shown that the KPS score is an independent factor that influences prognosis. Our analysis indicates that the addition of targeted drug therapy could reduce the importance of KPS scores. In other words, patients with very poor KPS scores who received targeted drug therapy could achieve a similar prognosis as patients with higher KPS scores who received cytotoxic chemotherapy. In addition, patients with good control of primary tumor, interval between initial treatment and BM greater than 1 year, presence of combined extracranial metastasis, the number of chemotherapy greater than 3 cycles, the lower Graded Prognostic Assessment class, presence of combined targeted drug therapy, adenocarcinoma, and higher cultural background had better prognosis. The presence of combined targeted drug therapy, the chemotherapy number greater than 3 cycles, and the cultural background are the independent prognostic factors. In the different cultural background groups, we can see that primary school plus average understanding of disease plus average family support is enough for NSCLC patients. There is no difference in prognosis between junior secondary school and senior secondary school group. Evidently, prospective clinical studies are still needed to determine more reasonable treatment regimes.

\section{Acknowledgments}

This study was supported by the Youth Project of Science and Technology of Shanghai (12ZR1448100); Scientific Research Foundation of Shanghai Pulmonary Hospital, Tongji University School of Medicine, Shanghai, People's Republic of China; and the Scientific Research Foundation of Shanghai Municipal Commission of Health and Family Planning (20154Y0147).

\section{Disclosure}

The authors report no conflicts of interest in this work.

\section{References}

1. David SS, Nasser KA, Claudia LH. Chapter 27: Cancer of the Lung: Section 2: Non-Small Cell Lung Cancer. In: Devita VT, Hellman S, Rosenberg SA, editors. Cancer: Principle and Practice of Oncology, 7th ed. Philadelphia, PA: Lippincott Williams \& Wilkins; 2004.

2. Chi A, Komaki R. Treatment of brain metastasis from lung cancer. Cancers (Basel). 2010;2(4):2100-2137.

3. Zabel A, Debus J. Treatment of brain metastases from non-small-cell lung cancer (NSCLC): radiotherapy. Lung Cancer. 2004;45 Suppl 2: S247-S252.

4. Chargari C, Pacaut C, Le Moulec S, et al. First assessment of whole-brain radiation therapy combined with pemetrexed-based chemotherapy in non-small-cell lung carcinoma: data on safety and efficacy. Anticancer Drugs. 2013;24(7):736-742.
5. Song Z, Zhang Y. Gefitinib and erlotinib for non-small cell lung cancer patients who fail to respond to radiotherapy for brain metastases. J Clin Neurosci. 2014;21(4):591-595.

6. Dinglin XX, Huang Y, Liu H, Zeng YD, Hou X, Chen LK. Pemetrexed and cisplatin combination with concurrent whole brain radiotherapy in patients with brain metastases of lung adenocarcinoma: a single-arm phase II clinical trial. J Neurooncol. 2013;112(3):461-466.

7. Kim JH, Kim HS, Kwon JH, et al. Systemic chemotherapy after cranial irradiation in patients with brain metastases from non-small cell lung cancer: a retrospective study. Lung Cancer. 2009;63(3):405-409.

8. Welsh JW, Komaki R, Amini A, et al. Phase II trial of erlotinib plus concurrent whole-brain radiation therapy for patients with brain metastases from non-small-cell lung cancer. J Clin Oncol. 2013;31(7):895-902.

9. Zeng YD, Zhang L, Liao H, et al. Gefitinib alone or with concomitant whole brain radiotherapy for patients with brain metastasis from nonsmall-cell lung cancer: a retrospective study. Asian Pac J Cancer Prev. 2012;13(3):909-914.

10. Stringhini S, Rousson V, Viswanathan B, Gedeon J, Paccaud F, Bovet P. Association of socioeconomic status with overall and cause specific mortality in the Republic of Seychelles: results from a cohort study in the African region. PLoS One. 2014;9(7):e102858.

11. Kontos E, Blake KD, Chou WY, Prestin A. Predictors of eHealth usage: insights on the digital divide from the Health Information National Trends Survey 2012. J Med Internet Res. 2014;16(7):e172.

12. Li J, Bentzen SM, Renschler M, Mehta MP. Regression after wholebrain radiation therapy for brain metastases correlates with survival and improved neurocognitive function. $J$ Clin Oncol. 2007;25(10): $1260-1266$.

13. Boogerd W, van der Sande JJ, van Zandwijk N. Teniposide sometimes effective in brain metastases from non-small cell lung cancer. $J \mathrm{Neu}$ rooncol. 1999;41(3):285-289.

14. Andrews DW, Scott CB, Sperduto PW, et al. Whole brain radiation therapy with or without stereotactic radiosurgery boost for patients with one to three brain metastases: phase III results of the RTOG 9508 randomised trial. Lancet. 2004;363:1665-1672.

15. Chang EL, Wefel JS, Hess KR, et al. Neurocognition in patients with brain metastases treated with radiosurgery or radiosurgery plus whole brain irradiation: a randomised controlled trial. Lancet Oncol. 2009;10:1037-1044.

16. Tallet AV, Azria D, Barlesi F, et al. Neurocognitive function impairment after whole brain radiotherapy for brain metastases: actual assessment. Radiat Oncol. 2012;7:77.

17. Li J, Bentzen SM, Renschler M, Mehta MP. Regression after wholebrain radiation therapy for brain metastases correlates with survival and improved neurocognitive function. J Clin Oncol. 2007;25: $1260-1266$.

18. Yamamoto M, Serizawa T, Shuto T, et al. Stereotactic radiosurgery for patients with multiple brain metastases (JLGK0901): a multiinstitutional prospective observational study. Lancet Oncol. 2014;15:387-395.

19. Heimberger AB, Learn CA, Archer GE, et al. Brain tumors in mice are susceptible to blockade of epidermal growth factor receptor (EGFR) with the oral, specific, EGFR-tyrosine kinase inhibitor ZD1839 (Iressa). Clin Cancer Res. 2002;8(11):3496-3502.

20. Hotta K, Kiura K, Ueoka H, et al. Effect of gefitinib ('Iressa', ZD1839) on brain metastases in patients with advanced non-small-cell lung cancer. Lung Cancer. 2004;46(2):255-261.

21. Ishida A, Kanoh K, Nishisaka T, et al. Gefitinib as a first line of therapy in non-small cell lung cancer with brain metastases. Intern Med. 2004; 43(8):718-720.

22. Cappuzzo F, Ardizzoni A, Soto-Parra H, et al. Epidermal growth factor receptor targeted therapy by ZD 1839 (Iressa) in patients with brain metastases from non-small cell lung cancer (NSCLC). Lung Cancer. 2003;41(2):227-231 


\section{Publish your work in this journal}

OncoTargets and Therapy is an international, peer-reviewed, open access journal focusing on the pathological basis of all cancers, potential targets for therapy and treatment protocols employed to improve the management of cancer patients. The journal also focuses on the impact of management programs and new therapeutic agents and protocols on
Dovepress

patient perspectives such as quality of life, adherence and satisfaction. The manuscript management system is completely online and includes a very quick and fair peer-review system, which is all easy to use. Visit http://www.dovepress.com/testimonials.php to read real quotes from published authors.

Submit your manuscript here: http://www.dovepress.com/oncotargets-and-therapy-journal 